\title{
La vaccination contre la paralysie contagieuse du porc à Madagascar
}

\author{
par P: BOURDIN, G. BUCK et H, JACOTOT
}

\section{INTRODUCTION}

La paralysie contagieuse du porc a commencé à sévir gravement à Madagascar au cours de la saison chaude 1945-1946. Depuis cette période, elle détermina sur le cheptel porcin de Madagascar une très forte mortalité, et amena une diminution de l'effectif porcin de l'Ile de 200.000 têtes entre 1941 et 1951 (1).

Le diagnostic expérimental du virus responsable de la maladie a été établi en 1950 par le Prof. P. Lépine et P. Atanasiu (2) à l'Institut Pasteur de Paris.

En 1951, le Prof. Verge de l'Ecole Nationale Vétérinaire d'Alfort a été chargé d'une mission d'étude concernant la maladie de Teschen à Madagascar et nous a guidé par ses conseils éclairés (3).

Les chercheurs du Laboratoire Central de l'Elevage de Madagascar se sont attachés à produire un vaccin préparé à partir du cerveau de porc afin de lutter contre cette redoutable épizootie. La production n'a pu être commencée qu'en 1953 avec des moyens précaires et dans des locaux trop petits mis très aimablement à notre disposition par $M$. le Directeur de l'Institut Pasteur de Tananarive.

A partir de 1955, la production a été en augmentant grâce à la construction d'un nouveau laboratoire et à l'acquisition d'un matériel important. Nous pensons pouvoir satisfaire bientôt les besoins en vaccin antiteschen de Madagascar par la préparation d'un vaccin sur cellules épithéliales rénales de porc.

$$
\text { ** }
$$

\section{VACCINATION DES PORCS AVEC UN VACCIN PREPARE SUR CERVEAU}

Le vaccin actuellement utilisé est un vaccin adsorbé sur gel d'alumine à 1 p. 100 selon la technique préconisée par Traub (4) puis phéniqué à 0,5 p. 100 .

\section{MATERIEL ET TECHNIQUE DE PREPARATION}

\section{Animaux.}

On utilise de jeunes porcelets sevrés de race locale.

\section{Technique d'inoculation.}

Les animaux sont inoculés par voie intracérébrale avec $1 / 4$ de $\mathrm{cm}^{3}$ de suspension virulente de centre nerveux provenant de porcelets purs inoculés avec la souche malgache S.I.C.E. diluée au $1 / 100$. Chaque animal reçoit 10.000 DL 50 de virus.

\section{Sacrification des animaux.}

Les porcelets inoculés sont surveillés matin et soir pendant 30 jours. Au bout du $8^{\mathrm{e}}$ jour, on note une élévation thermique et une perte de l'appétit, puis, rapidement, l'apparition de symptômes nerveux se traduisant par des mâchonnements avec hypersalivation, des vacillements des membres postérieurs, ou de la faiblesse du train antérieur. La paralysie totale peut apparaître d'emblée.

Si les symptômes observés sont douteux, il est procédé à un examen histologique du bulbe et de la moelle lombaire prélevés après sacrification de l'animal afin d'éliminer les centres nerveux des animaux atteints d'une autre affection.

\section{Sacrification des porcelets et récolte des cerveaux.}

Les animaux sont sacrifiés à la période de paralysie totale avec hyperthermie, car c'est 
à cette phase de la maladie que le cerveau est le plus riche en virus comme l'ont montré Buck et Serres (5).

Les centres nerveux sont prélevés aseptiquemcnt, récoltés dans un récipient stérile, pesés et congelés à $-30^{\circ}$.

\section{Préparation du vaccin.}

Les centres nerveux sont mis à décongeler pendant une nuit à la glacièrc à $+4^{0}$.

On pèse une certaine quantité de cerveau, on ajoute un poids égal d'eau distillée stérile et on effectue le broyage à l'aide d'un "mixer". Après cette opération, on porte la substance nerveuse dans un "Becher " et on ajoute de l'eau distillée, de telle façon que la substance soit diluée au $1 / 10$.

Cette suspension est mise dans un ballon contenant un égal volume de gel d'alumine à 2 p. $100 \mathrm{~d}$ 'extrait sec.

On agite pendant 2 heures pour bien homogénéiser; puis on ajoute l'acide phénique titré, nécessaire pour avoir 0,5 p. 100 d'acide phénique dans le vaccin. On pratique une nouvelle agitation de 30 minutes. Le vaccin est ensuite laissé à la température du laboratoire pendant 24 heures, puis mis à $+4^{\circ}$. Il est soumis à un contrôle bactériologique aérobie et anaérobie et à une épreuve d'innocuité avant son utilisation.

Le vaccin ainsi préparé conticnt :

5 p. 100 de substance cérébrale,

1 p. 100 d'extrait sec de gel d'alumine,

0,5 p. 100 d'acide phénique.

\section{Vaccination proprement dite.}

Les animaux sont vaccinés entre le $2^{\mathrm{e}}$ et le $3^{e}$ mois après la naissance par injection de $5 \mathrm{~cm}^{3}$ de vaccin par voie sous-cutanée en arrière de l'épaule. On fait deux injections à 15 jours d'intervalle. Il serait intéressant de faire une injection de rappel, 6 mois après la dernière injection, mais dans la pratique, cc rappcl ne peut être fait que dans les centres d'immunisation.

RESULTATS DE IA VACCINATION ANTITESCHEN A MADAGASCAR ENIRE LE 1er JANVIER 1956 ET LE 1ex JULLET 1957 (6)

\begin{tabular}{|c|c|c|c|}
\hline$\cdot$ & $\begin{array}{c}\text { Nombre } \\
\text { d'animaux vaccinés }\end{array}$ & $\begin{array}{l}\text { Nombre } \\
\text { d'animaux qui ont } \\
\text { contracté Ia maladio } \\
\text { après vacoination }\end{array}$ & $\begin{array}{l}\text { Pouroentage } \\
\text { des animaux ayant } \\
\text { oontraoté la maladie } \\
\text { après vaccination }\end{array}$ \\
\hline Province de TANANARIVE & 77.042 & 196 & $0,25 \%$ \\
\hline Province de FIANARANTSOA & 11.694 & 405 & $3,45 \%$ \\
\hline Province de MAJNGA & 3.946 & 40 & $1,01 \%$ \\
\hline Province de taMaTaVE : & & & . \\
\hline - Région cotièro & 204 & 114 & $55,8 \%$ \\
\hline - District d'Ambatondrazaka & 16.733 & 0 & $0 \%$ \\
\hline Province de TULEAR & & & $\cdot$ \\
\hline $\begin{array}{l}\text { (Les élevages de porcs sont } \\
\text { situés en régions côtières) }\end{array}$ & 2.175 & ${ }^{707}$ & $32,5 \%$ \\
\hline LABORATOIRE CENTRAL DE L'ELEVAGE' & 440 & 0 & 0 \\
\hline POUR L'ENSEMBLE DE MADAGASCAR & 112.234 & 1.462. & $1,30 \%$ \\
\hline
\end{tabular}

\section{Conclusion}

Dc cette ćtude statistiquc, il ressort que la vaccination anti-Teschen semble en échec dans les régions côtières. L'exemple de la province de Tamatave est typique. Sur la côte, le pourcentage des animaux atteints de maladie de
Teschen est de 55,8 p. 100, alors que dans le district d'Ambatondrazaka, situé à l'intérieur des terres et déjà à une certaine altitude, il a été pratiqué 16.733 vaccinations sans aucun échec.

De même dans la province de Tuléar, les 
élevages de porcs sont situés en région côtière et le pourcentage des porcs non immunisés est de 32,5 p. 100.

Pensant que le vaccin pouvait être tenu pour responsable de ces échecs, et même accusé de transmettre la paralysie contagieuse, car les accidents observés dans ces régions apparaissent le plus souvent à partir du $8^{*}$ jour après la première vaccination, nous avons contrôlé systématiquement l'innocuité du vaccin pour chaque lot préparé sur des porcelets de race pure qui sont les plus sensibles à la maladie. Sur 440 porcs vaccinés au Laboratoire, nous n'avons jamais observé un cas de paralysie contagieuse après vaccination. Les porcs vaccinés ont été ensuite éprouvés par voie intra-cérébrale avec une suspension virulente de substance nerveuse provenant de porcs inoculés avéc la souche malgache S.I.C.E. et récoltée à la période de paralysie complète avec hyperthermie. A cette période, la DL 50 calculée suivant la méthode de Reed et Muench (7) est de 10-6,6.

Pour l'épreuve, nous avons inoculé 10.000 $\mathrm{DL} 5020$ jours après la dernière vaccination, et nous avons gardé les porcs en observation pendant 30 jours :

Nombre de porcs vaccinés et éprouvés...$\ldots \ldots \ldots \ldots \ldots$.

Nombre de porcs morts après l'épreuve ................

Pourcentage des porcs non résistants à l'épreuve ............

15 p. 100

Pourcentage des porcs résistants. .

85 p. 100

Pour expliquer les accidents observés pendant la vaccination, nous devons insister sur le fait que la vaccination est pratiquée en milieu contaminé, et que les cas de maladie de Teschen apparaissent en moyenne vers le $8^{\mathrm{e}}$ jour après la première vaccination; assez souvent même ils apparaissent dans les premiers jours qui suivent la première vaccination et sont beaucoup plus rares dans les mois qui suivent la vaccination. En raison de ces faits, nous basant sur la durée de l'incubation de la maladie de Teschen qui est de 5 à 35 jours, et sur le contrôle systématique du vaccin au laboratoire, nous pensons que ces échecs ne sont pas imputables au vaccin mais dus à la présence du virus de Teschen dans l'organisme de l'animal avant la vaccination.

Nous avons observé des cas semblables au

$\left(^{*}\right)$ Ces animatx ont reçu 3 injections de $5 \mathrm{~cm}^{3}$ de vaccin à 10 juurs d' intervalle. laboratoire central de l'élevage et dans la province de Tananarive, où des lots d'animaux qui, par chance, n'avaient pas encore été vaccinés, présentaient des signes cliniques de maladie de Teschen. Sur ces lots d'animaux, nous avon's pu arrêter l'évolution de la maladie en injectant par voie sous-cutanée un immunsérum préparé sur le porc et limiter les pertes par paralysie contagieuse. Nous devons ajouter que le sérum n'a pas d'action thérapeutique sur les porcs atteints cliniquement.

Ces lots d'animaux ont été vaccinés 10 jours après l'injection du sérum sans présenter de signes cliniques de paralysie contagieuse.

Nous poursuivons actuellement nos recherches sur la séro-vaccination afin de voir si cette méthode est valable et si l'immunité conférée après séro-vaccination est aussi bonne que celle conférée par la vaccination simple.

\section{VACCINATION DES PORCELETS A PARTIR D'UN VACCIN PRÉPARÉ SUR CULTURE DE TISSUS}

\section{Culture du virus de Teschen sur cellules épithéliales de rein de porcelet en couche monocellulaire.}

La culture du virus de Teschen sur tissus a déjà été tentée par différents auteurs : Horstmann (8) en 1952 essaya de cultiver le virus de Teschen sur les fibroblastes de poulet et conclut que le virus semblait $y$ survivre et se multiplier. Larski (9) en 1955 obtint la culturc du virus de Teschen sur les cellules rénales de l'embryon de porcelet. Mayr et Schwobel (10) montrèrent en 1956 que le virus de Teschen pouvait se multiplier sur le tissu rénal de porcelet et obtinrent une action lytique du virus sur ces cellules à partir du $3^{\mathrm{e}}$ passage sur culture.

A Paris, dans le service des virus de l'Institut Pasteur, puis au Laboratoire Central de l'Elevage de Madagascar à Tananarive, P. Bourdin, P. Atanasiu, P. Lépine, $H$. Jacotot et $A$. Vallée (11) ont tenté de cultiver le virus de Teschen sur différents tissus. Nous avons essayé la culture sur les fibroblastes de l'embryon de poulet et navons pas obtenu de multiplication du virus, puis nous avons cultivé le virus sur le testiculc de porc et n'avons pas obtenu sa multiplication; enfin, en utilisant les cellules épithéliales du rein de porcelet, nous avons 
pu obtenir la multiplication du virus de Teschen sur ces cellules et l'apparition du pouvoir cytopathogène dès le deuxième passage.

Nous avons pu démontrer que l'agent responsable était le virus de Teschen en effectuant un test de séro-neutralisation sur tubes de culture (11).

\section{Test de séro-neutralisation.}

Nous avons mis 1.000 DL $50 \mathrm{du}$ virus de culture en présence d'une part d'un égal volume de différentes dilutions du sérum d'un porc hyperimmunisé contre la maladie de Teschen et d'autre part en présence du même volume de dilutions du sérum d'un jeune porcelet non vacciné. Finalement, nous avons 500 DL 50 du virus de culture en présence des dilutions de chacun des sérums.

Nous avons laissé les sérums et le virus en contact pendant une heure à la température du laboratoire, puis nous avons inoculé les tubes de culture à raison de 3 par dilution. Les tubes ont été placés dans une étuve à $37^{\circ}$ sur un portoir à tubes roulants et la lecture a été faite 5 jours après l'inoculation (voir tableau).

\section{Trypsine.}

Nous utilisons la qualité dite à $1: 300: 0,25$ p. 100 dissout dans une solution tamponnée type P.B.S. (12) mais sans chlorure de calcium ni chlorure de magnésium.

\section{Milieu de culture.}

Solution de Earle (13) contenant de l'hydrolysat : enzymatique de lactalbumine à raison de $5 \mathrm{~g}$ par litre $\ldots \ldots \ldots \ldots \ldots \ldots \ldots .950 \mathrm{~cm}^{3}$

Sérum de veau chauffé à $50^{\circ} \ldots .50 \mathrm{~cm}^{3}$. Solutions de vitamines B (14) (15). $1 \mathrm{~cm}^{3}$

\section{Antibiotiques.}

Pénicilline $40.000 \mathrm{U} / \mathrm{cm}^{3} \ldots \ldots \ldots \quad 2,5 \mathrm{~cm}^{3}$ Streptomycine $20.000 \mu \mathrm{g} / \mathrm{cm}^{3} \ldots . \quad 2,5 \mathrm{~cm}^{3}$

\section{Technique de culture.}

Nous pratiquons la technique de culture de tissu en couche monocellulaire après trypsinisation mise au point par Dulbecco et Vogt (12), puis modifiée par Youngner (16).

\begin{tabular}{|c|c|c|c|c|c|c|c|c|c|}
\hline Dilutions & $\frac{1}{32}$ & $\frac{1}{64}$ & $\frac{1}{128}$ & $\frac{1}{256}$ & $\frac{1}{512}$ & $\frac{1}{1024}$ & $\frac{1}{2048}$ & $\frac{1}{4096}$ & $\frac{1}{8192}$ \\
\hline Cellules non dégénérées & 3 & 3 & 3 & 3 & 3 & 3 & 3 & 3 & 2 \\
\hline Cellules dégénérées & 0 & 0 & 0 & 0 & 0 & 0 & 0 & 0 & 1 \\
\hline
\end{tabular}

SERUM NORMAL

\begin{tabular}{|c|c|c|c|c|c|c|}
\hline Dilutions & $\frac{1}{4}$ & $\frac{1}{8}$ & $\frac{1}{16}$ & $\frac{1}{32}$ & $\frac{1}{64}$ & $\frac{1}{128}$ \\
\hline Cellules non dégénérées & 3 & 0 & 0 & 0 & 0 & 0 \\
Celliules dégénérées & 0 & 3 & 3 & 3 & 3 & 3 \\
\hline
\end{tabular}

TECHNIQUE DE PREPARATION

DU VACCIN CULTURE

\section{Animaux.}

Nous prélevons les reins sur des porcelets âgés de 20 à 30 jours.
Les porcelets sont saignés, puis les reins prélevés aseptiquement sont décapsulés, la zone corticale est prélevée, mâchée à l'aide d'une pince de Kocher, puis finement hachée à l'aide d'une lame de rasoir. Les fragments tissulaires sont introduits dans une fiole de Fourneau contenant un barreau aimanté où ils sont lavés 
plusieurs fois à l'aide du P.B.S. incomplet.

Après le dernier lavage, on introduit environ $50 \mathrm{~cm}^{3}$ de la solution de trypsine préalablement portée à $37^{\circ}$, la fiole de Fourneau est posée sur un agitateur magnétique placé dans une étuve à $37^{\circ}$. L'agitateur est mis en route à vitesse réduite; au bout de vingt minutes, le liquide surnageant est récolté après décantation. Les trois premières récoltes sont éliminées, car elles ne contiennent pas de cellules. Les récoltes suivantes sont recueillies directement dans un pot à centrifuger de $250 \mathrm{~cm}^{3}$ maintenu à la température de la glace fondante.

La durée de la digestion varie avec les animaux : en moyenne il faut cinq heures pour digérer 8 reins de porcelets.

La suspension cellulaire est centrifugée à $1.000 \mathrm{t} / \mathrm{mn}$; le liquide surnageant est éliminé, puis remplacé par du P.B.S. incomplet dans lequel nous remettons les celulles en suspension par une agitation vigoureuse. Nous effectuons cette opération trois fois de suite.

Les cellules, après la dernière centrifugation, sont introduites dans le milieu de culture à raison de $1 \mathrm{~cm}^{3}$ de culot cellulaire pour $100 \mathrm{~cm}^{3}$ de milieu. Nous obtenons ainsi environ 500.000 cellules épithéliales par $\mathrm{cm}^{3}$ de milieu. Un rein de porc donne environ $25 \mathrm{~cm}^{3}$ de culot cellulaire et nous préparons ainsi $2.500 \mathrm{~cm}^{3}$ de culture.

Le milieu de culture est réparti dans des boîtes de Roux de $1.000 \mathrm{~cm}^{3}$ à raison de $100 \mathrm{~cm}^{3}$ de milieu par boîte. Ces boîtes sont bouchées à l'aide de bouchons de caoutchouc gris et mises à l'étuve à $37^{\circ}$ et on obtient une couche monocellulaire complète en cinq à sept jours, suivant l'âge des porcelets utilisés.

\section{Inoculation du virus.}

Nous éliminons le milieu de culture des flacons et introduisons dans chaque flacon $10 \mathrm{~cm}^{3}$ de virus de culture. Les flacons sont posés à plat, la couche cellulaire tournée vers le bas, et nous laissons le virus en contact avec les cellules pendant 1 heure. Après ce délai, nous introduisons $190 \mathrm{~cm}^{3}$ de milieu contenant seulement 2 p. 100 de sérum de veau chauffé à $50^{\circ}$. Les flacons sont bouchés à l'aide d'un bouchon de caoutchouc et placés dans une étuve à $370^{\circ}$. Dès le deuxième jour après l'inoculation, les cellules rénales commencent à se lyser et le troisième jour elles sont complètement dégénérées. Nous récoltons le liquide virulent et pratiquons un contrôle bactériologique et un titrage sur tubes de culture et sur porcelets.

\section{Titrage sur tubes de culture.}

Nous pratiquons des dilutions sériées du virus allant de $10^{-1}$ à $10^{-7}$. Ces dilutions sont faites dans de l'eau bidistillée. Nous inoculons 3 tubes de culture par dilution à raison de $0,3 \mathrm{~cm}^{3}$. Le virus est laissé en contact une heure avec les cellules, puis nous ajoutons le milieu et portons les tubes de culture dans un portoir à tubes roulants. La lecture est faite entre le $2^{\mathrm{e}}$ et le $5^{\mathrm{e}}$ jour après l'inoculation. Nous calculons la DL 50 suivant la méthode de Reed et Muench et nous trouvons en moyenne DL 50 égale ou supérieure à $100^{6}$.

\section{Technique de préparation du vaccin.}

Nous utilisons un vaccin aluminé dont la préparation dérive de la technique de Traub (4).

Le liquide de culture est mis en contact avec un volume égal de gel d'alumine à 2 p. 100 d'extrait sec. De cette façon, la quantité finale de gel d'alumine dans le vaccin est de 1 p. 100. Le mélange est agité pendant une demi-heure, puis réparti en flacons et conservé à la glacière à $+4^{\circ}$. Nous effectuons un contrôle bactériologiquc et une épreuve d'innocuité sur porcelet qui est complétée par une épreuve d'efficacité.

\section{RESULTAT'S OBTENUS \\ AVEC LE VACCIN DE CULTURE}

\section{Choix des animaux.}

Nous utilisons des jeunes porcelets de race pure âgés de 45 à 60 jours, les animaux de race pure étant plus sensibles à la paralysie contagieuse que les porcs locaux.

\section{Technique de vaccination.}

Les animaux sont vaccinés par voie souscutanée. Nous injectons $5 \mathrm{~cm}^{3}$ de vaccin en arrière de l'épaule, faisons 3 injections à 10 jours d'intervalle, et vingt jours après la dernière injection, les animaux sont éprouvés par voie intracérébrale avec $10.000 \mathrm{DL} 50$ de virus issu de cerveau de porcs inoculés avec la souche S.I.C.E. Les porcs sont observés pendant 30 jours après l'épreuve.

\section{Résultat.}

Nos expériences ont porté sur 120 porcelets : Nombre de porcs vaccinés .... 120

Nombre de morts après épreuve 9

Pourcentage des pertes....... 7,5 p. 100

Pourcentage des résistants .... 92,5 p. 100 


\section{CONCLUSION}

La vaccination des porcelets contre la maladie de Teschen à l'aide d'un vaccin préparé à partir des centres nerveux des porcelets a permis de lutter avec efficacité contre la paralysie contagieuse du porc à Madagascar.

Le virus de Teschen peut se multiplier sur les cellules épithéliales rénales du porcelet, il est doué du pouvoir cytopathogène pour ces cellules.

Le milieu de culture récolté après la dégénérescence totale des cellules peut être utilisé pour la préparation d'un vaccin qui est doué d'un bon pouvoir immunisant à l'égard de la paralysie contagieuse du porc.

(Laboratoire Central de l'Elevage et des Epizooties de Madagascar.)

\section{BIBLIOGRAPHIE}

1. Buck (G.) et Quesnel (J.-J.). - Bull. Bureau Interafr. Epiz., 1954, 2, 326.

2. LÉPine (P.) et Atanasiu (P.). - Ann. Inst. Pasteur, 1950, 79, 113.

3. Verge (J.).-Encycl.Vét.Périod., 1950,11,366.

4. Traub (E.). - Archiv. für Tierheilk., 1941, 77, 52 .
5. Buck (G.) et Serres (H.). - Ann. Inst. Pasteur, 1957, 93, 230.

6. Lalanne (A.). - Bull. inter. Epiz. (sous presse).

7. ReEd et Muench. - Ann. J. Hyg., 1938, 28, 493.

8. HoRstmann. - J. immunolog., 1952, 69, 379.

9. LarsKI. - Méd. Vét. (Varsovie), 1955. 11, 589.

10. MaYr (A.) et Schwobel (W.). - Mh. Tierheilk, 1956, 8, 49.

11. Bourdin (P.), Atanasiu (P.), Lépine (P.), JACOTOT (H.) et VALLÉE (A.). - Ann. Inst. Pasteur, 1957, 93, 581.

12. Dulbecco (R.) et Vogt. - J. Exp. Méd., 1954, 99, 167.

13. Earle. - J. Nat. Cancer. Inst.; 1943, 90, 654.

14. Eagle (H.). - Proc. Soc. Exp. Biol., 1955, $89,96$.

15. LéPine (P.), Slizewicz (P.), Daniel (Ph.) et Pacaud (M.). - Ann. Inst. Pasteur, 1956, 90, 654.

16. Youngner (J.-S.). - Proc. Soc. Exp. Biol., 1954, 85, 202.

\section{SUMMARY}

\section{Anti-Teschen disease vaccination in Madagascar}

1. The vaccine substance in use at present consists of brain tissue of young pigs of local breeds inoculated intracerebrally and sacrificed at the period in the syndrome when paraplegia is complete and hyperpyrexia is still present. This tissue is adsorbed on aluminium gel at 1 per cent and then carbolized at 0,5 per cent.

Piglets are vaccinated at between 2 and 3 months of age by two subcut. injections each of $5 \mathrm{ml}$ at 15 days interval. During the period lst Junuary 1956 - lst July 1957, 112,234 pigs have been vaccinated of which only 1,3 per cent subsequently contracted the infection.

2. The authors also describe the tissue culture of this virus on monocellular layers of pig kidney cells and from this culture the preparation of an aluminium - gel vaccine. It is hoped that this method of preparation will satisfy the heavy demand for anti-teschen vaccine.

\section{RESUMEN}

\section{La vacunación contra la paralisis contagiosa del cerdo en Madagascar.}

1) La vacuna actualmente utilizada es una vacuna adsorbida por un gel de aluminio al 1 por 100 , luego fenicada al 0,5 por 100 , que se prepara a partir de lechones de vaza local inoculados por via intracerebral y sacrificados en el periodo de paralisis total con hipertermia. Los cerdos son vacunados entre el segundo y tercer mes con dos inyecciones subcutaneas de $5 \mathrm{ml}$ cada una y con 15 dias de intervalo. 112234 cerdos han sido vacunados desde el $1^{\circ}$ de Enero de 1956 al $1^{\circ}$ de Julio de 1957 , de los cuales el 1,3 por 100 han contraido la enfermedad despues de la vacunación.

2) Los autores deseriben la tecnica de cultivo del virus de Teschen en celulas epiteliales de riñon de lechón en capa monocelular, y a partir de este cultivo la preparación de una vacuna al aluminio: Piensan ellos poder satisfacer la demanda de vacuna antiteschen. 